\title{
Hepatitis B: situación mundial y regional
}

\author{
Gina Tambini', KS Suan Mung ${ }^{2}$, Jorge Raad ${ }^{3}$
}

\begin{abstract}
Resumen
Los autores, hacen un breve resumen de la importancia de la hepatitis $\mathrm{B}$, haciendo énfasis en los panoramas mundial y regional, identificando luego las acciones adoptadas a nivel continental para lograr la erradicación de la hepatitis B. Describen además las implicaciones económicas y la vigilancia epidemiológica de la enfermedad. el artículo incluye un análisis de debilidades, oportunidades, fortalezas y amenazas, DOFA del programa del control de la hepatitis en america.
\end{abstract}

\section{Hepatitis B: world and regional situation}

This is a short article about the Hepatitis B erradication programme in the Americas, particular emphasis being placed on world and continental panoramas, followed by identification of activities adopted at continental level to achieve its erradication. The implications of the economic and epidemiological surveillance aspects of hepatitis $B$ are presented. The American hepatitis control programme's weaknesses, opportunities, strengths and menaces are also analysed.

La hepatitis viral es una enfermedad en cuya etiología se han identificado, por lo menos, seis virus diferentes, los cuales pueden infectar al hombre desde su período neonatal hasta la vejez. El virus causa una extensa lesión hepática que tiene muchas consideraciones fisiopatológicas, dependiendo del agente causal y de las variables dependientes del paciente, ya sea que la infección se suceda en la niñez, en la juventud o en el estado adulto.

La investigación epidemiológica ha proporcionado una serie de datos útiles que han permitido detectar los grupos de alto riesgo y las posibles medidas para prevenir cada una de las hepatitis.

El mecanismo de transmisión mediante el contacto sexual del virus $B$ de la hepatitis abrió un nuevo panorama en la epidemiología de estas enfermedades, a lo cual se ha sumado que pueden existir otras vías de infección diferentes a las tradicionalmente conocidas para los virus de las hepatitis.

Las diferentes rutas de infección que se conocen en la actualidad para cada uno de los virus comprometidos en la hepatitis hacen que las posibilidades de infección aumenten en la medi-

Médica y Magister en Salud Pública, Organización Panamericana de la Salud, representación en Colombia.

2 Médico y Magister en Salud Pública.

${ }^{3}$ Médico, Subdirector de Epidemiología y Laboratorio Nacional de Referencia, Instituto Nacional de Salud de Colombia, Santa Fe de Bogotá; Profesor Titular, Universidad de Caldas. Manizales.

Recibido para su publicación: 10 de noviembre de 1997. - Aprobado para su publicación: 6 de junio de 1998. 
da que los seres humanos avanzan en su edad cronológica.

Las manifestaciones clínicas y los estudios generales de laboratorio no son por sí solos capaces de diferenciar una hepatitis viral de otra. Es necesario identificar los diferentes marcadores virales que caracterizan a cada uno de los virus.

\section{Panorama mundial}

Cada año, cerca de un millón de personas mueren a causa de la infección aguda o crónica por hepatitis $\mathrm{B}$, considerándose una de las mayores causas de morbilidad y mortalidad en humanos.

Mundialmente, se estima que más de 350 millones de personas están infectadas crónicamente con el virus de la hepatitis $B$ (VHB) y 2 billones de personas tienen marcadores serológicos positivos lo cual indica infección actual o pasada con el virus. Tres cuartos de la población mundial vive en áreas con niveles altos de infección.

El VHB causa el $83 \%$ de los 540.000 nuevos casos de cáncer de hígado en el mundo, que es una de las tres primeras causas de muerte por cáncer en hombres en el este y sudeste asiático, la cuenca del Pacífico y Africa subSahara.

La vacuna hace parte de la inmunización rutinaria en 81 países.

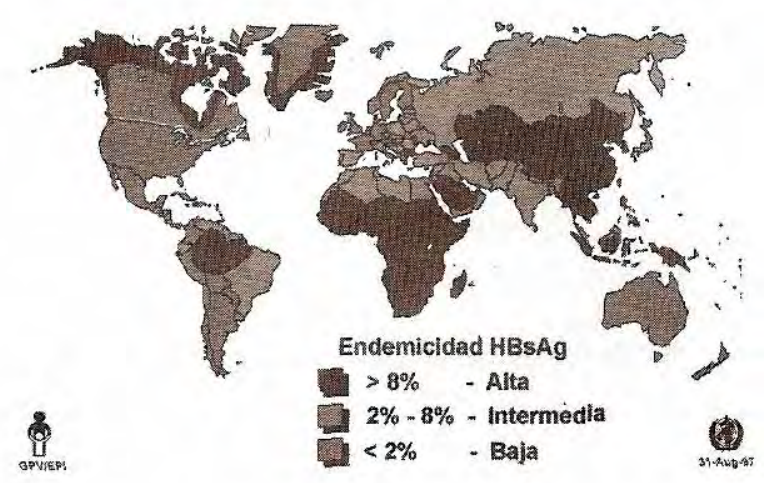

Figura 1. Prevalencia de la hepatitis B en el mundo (2).

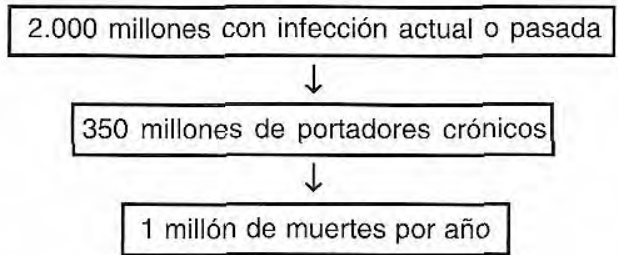

Figura 2. Panorama mundial de la hepatitis $\mathrm{B}$ - número de infecciones, portadores crónicos y muertes.

Cuadro 1. Hepatitis B: portadores crónicos en regiones endémicas.

\begin{tabular}{|c|c|c|c|c|}
\hline Región & $\begin{array}{l}\text { Población } \\
\text { (millones) }\end{array}$ & $\begin{array}{c}n \text { VHB portadiores } \\
\text { (millones) }\end{array}$ & $\%$ & $\begin{array}{l}\text { VHB } \\
\text { portadores }\end{array}$ \\
\hline Africa & 413 & 49,5 & & 17,5 \\
\hline $\begin{array}{l}\text { Asia } \\
\text { Medio }\end{array}$ & 2.757 & 220,0 & & 77,0 \\
\hline Oriente & 191 & 7,6 & & 3,0 \\
\hline $\begin{array}{l}\text { Latino- } \\
\text { américa }\end{array}$ & 410 & 6,6 & & 2,3 \\
\hline Oceanía & 6 & 0,6 & & 0,2 \\
\hline Total & 3.777 & 284,3 & & 100,0 \\
\hline
\end{tabular}

\section{Panorama regional}

Se ha estimado que 140.000 a 400.000 nuevos casos de hepatitis B pueden estar ocurriendo anualmente en Latinoamérica, dos tercios de ellos en Suramérica, incluyendo 440 a 1.000 casos de hepatitis fulminante.

La epidemiología de la infección por el VHB varía grandemente según la localización geográfica y los factores sociodemográficos. Mientras es cierto que las infecciones con el VHB y VHD (virus de hepatitis delta) son altamente prevalentes en Suramérica, existen notorias diferencias regionales en las tasas de infección y de portadores crónicos, no solo entre las diferentes naciones en la región sino también dentro de los países individualmente.

Algunas de las áreas con alta endemicidad son la cuenca amazónica y el borde norte del continente.

Información suficiente está disponible para formular estrategias claras y precisas que guíen hacia la prevención, el control y hasta la erradicación del VHB en Latinoamérica.

Debido a la gran carga que significa la deuda externa en muchos de los países de 
Latinoamérica, las consideraciones de costoefectividad serán de suma importancia.

Muchos estudios de seroprevalencia del VHB llevados a cabo en mujeres gestantes en áreas urbanas de Suramérica, no han podido demostrar claramente el patrón de la transmisión perinatal. Así mismo, en contraste con los hallazgos en poblaciones asiáticas, el antígeno e de la hepatitis $B(\mathrm{HBeAg})$ es positivo en baja frecuencia en mujeres portadoras del HBsAg (antígeno de superficie) y el ADN-VHB es también negativo cuando se determina por las técnicas de PCR, sugiriendo una baja tasa de transmisión perinatal en esta población.

El resultado de muchos estudios seroepidemiológicos sugiere que, en regiones hiperendémicas, las cohortes de portadores crónicos del VHB probablemente surgen por transmisión perinatal o por infección en la niñez temprana.

Estudios realizados en poblaciones urbanas con bajas tasas de portadores crónicos del VHB sugieren que la transmisión sexual es la forma predominante como se disemina el VHB en esas áreas y que los sujetos positivos al VHB son más frecuentemente adultos que niños. Sin embargo, algunos países han informado una alta prevalencia de infección por el VHB en las áreas urbanas pobres o en segmentos de población de baja condición socioeconómica. Generalmente, el resultado de varias investigaciones retrospectivas o prospectivas llevadas a cabo sugieren que, probablemente, la transmisión horizontal representa la mayor forma de disemi- nación del VHB en las ciudades de Latinoamérica. Sin ninguna duda, la prevalencia del VHB reportada en áreas urbanas y en poblaciones sanas es baja en comparación a la observada en poblaciones aborígenes.

Los grupos de población con alto riesgo incluyen aquellos con múltiples parejas sexuales, los hombres homosexuales, los drogadictos, los familiares de los portadores crónicos del VHB, los recién nacidos de madres con infección activa, el personal médico que está expuesto a contaminación por sangre o productos sanguíneos, los inmigrantes de áreas endémicas del VHB y los compañeros sexuales de la población incluida en alto riesgo. Estudios epidemiológicos han documentado infección por VHB en más del $50 \%$ de los miembros de los grupos de riesgo.

\section{Acciones tomadas}

La vacuna contra la hepatitis $B$ está disponible desde 1982 y ha sido utilizada en cientos de millones de individuos con excelentes resultados de seguridad e impacto en la enfermedad. Durante los últimos años, el precio de la vacuna ha disminuido, hasta el punto que es factible su amplio uso en programas de inmunización infantil.

Los portadores del VHB han sido reducidos de altas a bajas prevalencias en cohortes de niños inmunizados en muchos países. El seguimiento de las cohortes de niños inmunizados por 10 a 15 años ahora confirma que la prevalencia de

Tabla 2. Vacunación contra la Hepatitis B - Situación mundial (2).

\begin{tabular}{lccccc}
\hline Región & No. de países & $\begin{array}{c}\text { Inmunización } \\
\text { infantil }\end{array}$ & $\begin{array}{c}\text { Inmunización en } \\
\text { adolescentes }\end{array}$ & $\begin{array}{c}\text { En planeación } \\
\text { Países que reportan } \\
\text { coberturas }\end{array}$ \\
\hline $\begin{array}{l}\text { Africa } \\
\text { América }\end{array}$ & 47 & 6 & 0 & 3 & 2 \\
$\begin{array}{l}\text { Mediterráneo } \\
\text { Oriental }\end{array}$ & 40 & 10 & 2 & 2 & 1 \\
$\begin{array}{l}\text { Europa } \\
\text { Asia sudeste }\end{array}$ & 23 & 15 & 0 & 0 & 13 \\
$\begin{array}{l}\text { Pacífico } \\
\text { Occidental }\end{array}$ & 49 & 13 & 8 & 7 & 12 \\
** Basado en reportes recibidos por OMS a Septiembre 1996. & 5 & 0 & 2 & 3 \\
\end{tabular}


Cuadro 1. Recomendaciones del Grupo Asesor Mundial del Programa Ampliado de Inmunizaciones, 1991, que fue aprobado por la Asamblea Mundial en 1992.

1. La vacuna contra la hepatitis $B$ deberá se rintegrada en los programas nacionales en todos los países con prevalencia de portadores crónicos del VHB (HBsAG) el $8 \%$ o más para 1995, y en todos los paises para 1997.

2. Grupo objeto y estrategias pueden varias con la epidemiología local de la enfermedad.

3.Cuando la prevalencia de portadores crónicos es igual o mayor de $2 \%$ la más efectiva estrategia es la incorporación de la vacuna contra el VHB en los esquemas de vacunación.

4.Países con prevalencia baja deben considerar la vacunación de todos los adlecentes como adición o alternativa a la inmunización infantil.

Fuente: WHO, Expanded Program on Immunization; Hepatitis B Vaccine - making global progress; Update, October 1996

portadores del VHB ha sido reducida de 10-15\% (alta endemicidad), niveles previos a la vacunación, a menos de $2 \%$ en Asia y a menos de $1 \%$ en otras áreas del mundo.

Cerca de 81 países (39\%) han introducido la vacuna en sus programas regulares de vacunación; sin embargo, algunos todavía no han logrado coberturas óptimas. Cincuenta y ocho de 81 países $(72 \%)$ reportan coberturas a OMS y 31 de ellos (53\%) reportan coberturas iguales o mayores a $80 \%$. Esto significa que muchos de los niños que están en riesgo de infección por hepatitis B no están recibiendo la vacuna actualmente.

Actualmente, de los 40 países en América, 10 han integrado la vacunación contra la hepatitis $B$ en sus esquemas de vacunación. Ellos son: EUA, Canadá, Cuba, Guayana Francesa, Guadalupe, Martinique, Puerto Rico, Saint Keith and Nevis, Islas Vírgenes y Colombia. Dos países (Brasil y Perú) están planeando la integración de la vacuna contra la hepatitis $B$ en sus esquemas de vacunación.

Por lo menos, dos estrategias de vacunación universal se han considerado: la vacunación regular de los niños y la vacunación regular de los adolescentes. Aunque la vacunación regular de los adolescentes podría tener un impacto más rápido en la incidencia de la hepatitis $\mathrm{B}$, la vacunación regular de los niños es la estrategia preferible en la mayoría de los países con baja endemicidad por varias razones.

Los programas nacionales de vacunación en niños, que son parte del PAI, son altamente efectivos para la distribución y administración de la vacuna a los niños y están muy bien establecidos en casi todos los países. La vacunación con las vacunas convencionales del programa alcanza $80 \%$ de cobertura en la población infantil del mundo. La integración de la vacunación contra el VHB en el sistema del PAI es una forma costo-efectiva de alcanzar la inmunización universal.

La vacunación en adolescentes alcanzará el más rápido impacto en la incidencia de la infección por VHB en los países donde predominantemente la transmisión se presenta en adultos.

Con el objetivo de optimizar los recursos limitados y lograr un programa costo-efectivo a corto plazo, es recomendable ubicar recursos (humanos y financieros) en las áreas de alto riesgo (áreas con alta endemicidad-prevalencia de HBsAg igual o mayor a $8 \%$ ).

\section{Identificación de áreas de alto riesgo}

Como se describió anteriormente, en las áreas hiperendémicas, la infección por el VHB se origina por transmisión perinatal o en la niñez temprana. Cuando la infección por el VHB ocurre cerca del nacimiento, se estima que 70 a $90 \%$ serán portadores crónicos, entre ellos 30 a $50 \%$ desarrollará enfermedad hepática crónica que se desencadenará en muerte por cirrosis o cáncer hepático primario. Por tanto, los más jóvenes están en más alto riesgo.

Para identificar las áreas de riesgo se puede considerar la siguiente información disponible en los países:

- del Sistema Nacional de Información: datos de morbilidad y mortalidad de cirrosis o cáncer hepático por división geopolítica; 
Cuadro 3. El riesgo de ser portador está relacionado con la edad de la infección (2).

\begin{tabular}{cc}
\hline Edad de la infección & Riesgo de ser portador (\%) \\
\hline Nacimiento & 90 \\
$1-6$ meses & 80 \\
$7-12$ meses & 60 \\
$1-4$ años & 35 \\
Adultos & 10 \\
\hline
\end{tabular}

- estudios especiales seroepidemiológicos para determinar prevalencia de infección y portadores crónicos, y modos de transmisión de la enfermedad;

- bancos de sangre (determinación de HBsAg en donantes de sangre).

La mayoría de la información de los sistemas nacionales está basada en el diagnóstico clínico sin confirmación serológica. Los diagnósticos inadecuados de hepatitis por suero (en lugar de hepatitis B) o hepatitis infecciosa (en lugar de hepatitis A) se reportan en el sistema.

La información de los bancos de sangre constituye una fuente de información clave con relación a la identificación de áreas de alta endemicidad. Sin embargo, calcular tasas de prevalencia tiene limitaciones inherentes ya que la población de donantes es en su mayoría mayores de 18 años, constituyen una muestra selecta en la cual los enfermos no están incluidos, usualmente los bancos de sangre están ubicados en áreas urbanas y están equipados para desarrollar las pruebas serológicas para detectar los marcadores de hepatitis B.

\section{Implicaciones económicas}

Actualmente, la vacuna contra hepatitis $B$ derivada de plasma puede ser obtenida a US $\$ 0,50$ 1,00 por dosis pediátrica en países en desarroIlo. En competencia, el precio de la vacuna recombinante para países en desarrollo ha disminuido, pero, todavía es algo más costosa que la vacuna derivada de plasma. Sin embargo, a US $\$ 0,50$ por dosis, las tres dosis necesarias cuestan más que las otras seis vacunas del PAI (i.e., BCG, 3 dosis de DPT, 4 dosis de VOP y antisarampión).

Para muchos países, el mayor impedimento para la introducción universal de la vacuna con- tra la hepatitis $B$ ha sido el costo de la vacuna. Muchos países con alta prevalencia de portadores crónicos y altas coberturas de vacunación están utilizando la vacuna; sin embargo, en los países pobres, aquellos con el ingreso per capita de US\$500, la vacuna generalmente no está disponible.

En países con áreas hiperendémicas, el costoefectividad del programa será naturalmente mayor cuando los recursos se asignen a las áreas de alta prevalencia como prioridad.

\section{Vigilancia epidemiológica}

Debido a que la infección por el VHB es generalmente asintomático en niños pequeños, la vigilancia de la enfermedad limita su papel especialmente al seguimiento del programa de vacunación. En su lugar, el programa de vacunación debe monitorizarse a través de indicadores de proceso y de estudios de infección asintomática de hepatitis $\mathrm{B}$ (i.e., estudios serológicos).

Se debe considerar la realización de estudios de seroprevalencia en varias áreas para determinar los niveles basales de prevalencia de infección y de portadores crónicos para que, después de implementada la vacunación, se determine el impacto del programa.

Se debe considerar el establecimiento de un sistema de vigilancia de todas las hepatitis con el objetivo de determinar la etiología de la hepatitis y determinar los factores de riesgo de la enfermedad. Puesto que el apoyo del laboratorio es necesario para determinar los diferentes tipos de hepatitis y que los recursos son limitados para la compra de los reactivos para mantener un sistema de vigilancia nacional, se debe considerar el establecer un sistema de vigilancia centinela. En el sistema centinela, sólo los casos captados en áreas definidas son evaluados con pruebas de laboratorio y encuestados con relación a factores de riesgo de hepatitis. Este enfoque puede proveer bases para futuros esfuerzos de utilizar la vigilancia para evaluar el impacto del programa.

Al realizar el análisis DOFA del programa de control de la hepatitis $B$, en la región de las Américas se observa: 


\section{Debilidades}

La población objeto es amplia (recién nacidos, niños, adolescentes y adultos).

El precio de las tres dosis de vacuna contra hepatitis B es menor que las vacunas del PAI en uso actual.

\section{Oportunidades}

El compromiso político con el programa de inmunizaciones está en ascenso en la mayoría de países.

\section{Fortalezas}

Los programas de vacunación nacional, que son parte de la red del PAI, están establecidos en los países y son altamente eficientes para la distribución y administración de las vacunas.

Se tiene acceso a una vacuna contra la hepatitis B segura, efectiva y el costo está en descenso.

\section{Amenazas}

La competición de recursos con otras enfermedades que impactan más por las características de la fisiopatología de la infección, la morbimortalidad y las connotaciones sociales (i.e., HIV-SIDA).

\section{Conclusión}

La hepatitis $B$, entre las enfermedades transmisibles, debe ser considerada como una enfermedad a la cual los esfuerzos de prevención y control deberían asignarle una alta prioridad por las muertes e incapacidades que produce y considerando la existencia de una vacuna contra hepatitis B, segura, con alta eficacia y de costo en descenso. A medida que los países van fortaleciendo sus programas nacionales de vacunación, se deben realizar esfuerzos para que la vacunación contra la hepatitis $B$ sea universal.

\section{Referencias}

1. Hollinger B. Hepatitis $B$ virus. In: Fields BN, Knipe DM, Howley PM, editors. Virology. Philadelphia: Lippincott-Raven; 1996. p.2739-42.

2. WHO. Expanded Program on Immunization. Hepatitis B vaccine: making global progress. Update, October 1996.

3. Bloom BS, Hillman AL, Fendrick A, Schwartz M, Sanford J. A reappraisal of hepatitis B virus vaccination strategies using cost-effectiveness analysis. Ann Intern Med 1993;118(4):298-306.

4. Fay $\mathbf{O H}$, Latin American Study Group. Hepatitis B in Latin America: epidemiological patterns and eradication strategy. Vaccine 1990;8(Suppl.): S100S106.

5. Torres JR. Hepatitis B and hepatitis delta virus infection in South America. Gut 1996;38(5)(Suppl.): 48S-55S.

6. Kane MA. Progress on the control of hepatitis B infections through immunization. Gut 1993; (Suppl.): S10-S12.

7. Pia G, Pierre VD. Prevention and control of hepatitis $\mathrm{B}$ in the community. Communicable Diseases Series, No. 1. Belgium: Jacqmain Ltd.; 1996.

8. Shapiro CN, Goldstein ST. Prevention and control of hepatitis B in Peru. Report of a USAID consultation. November, 1996.

9. Maynard JE. Hepatitis B: global importance and need for control. Vaccine 1990;8(Suppl.):S18-S20.

10. WHO. Global programme for vaccines and immunization. Programme report 1996. WHO/GPV/97.01, Geneve, May, 1997.

11. WHO. World Health Report 1997. Vaccines against aancer. Geneve: WHO, 1997.

12. Koff RS. Hepatitis B and hepatitis D. In: Gorbach SI, Bartlett JG, Blaklow NR, editors. Infectious diseases. 2th ed. Philadelphia: Saunders; 1998. p.850-8. 


\section{Instrucciones para los autores}

Biomédica es la revista del Instituto Nacional de Salud. Es una publicación trimestral, eminentemente científica.

Está amparada por la resolución No. 03768 de 1981, emanada del Ministerio de Gobierno.

Ninguna publicación, nacional o foránea, podrá reproducir o traducir sus artículos o resúmenes, sin previa autorización escrita del editor.

$\mathrm{Ni}$ la revista, ni el instituto asumen responsabilidad alguna por los puntos de vista expresados por los autores.

La revista no publicará ningún tipo de propaganda comercial. Los nombres de equipos, materiales y productos manufacturados que eventualmente puedan mencionarse, no implican recomendación o propaganda para su uso y sólo se mencionarán como identificación genérica.

1. Biomédica: acepta para publicación artículos, fruto de la investigación, que contribuyan a ampliar los conocimientos sobre biomedicina, realizados tanto en el Instituto Nacional de Salud como en cualquier otro centro de investigación.

2. Biomédica publicará la siguiente categoría de trabajos:

a. Artículos originales: son trabajos inéditos derivados de una investigación biomédica que arroja información nueva sobre aspectos específicos y contribuye de manera relevante al conocimiento y estructuración del movimiento científico del país. Se exceptúan del carácter inédito, aquellos trabajos de investigadores del instituto que hayan sido ya publicados en revistas extranjeras de reconocida prestancia, previo consentimiento escrito del autor y de la respectiva revista.

b. Artículos de investigación básica aplicada: son trabajos de investigación básica que, sin ser originales, utilizan una metodología científica en la confirmación de trabajos previos o introducen modificaciones en procedimientos técnicos.

c. Artículos de investigación clínica: son trabajos de investigación que, utilizando una metodología científica, informan sobre casuística, epidemiología, tratamiento, sistemas diagnósticos, y que pueden ser de interés para el médico en ejercicio.

d. Artículos de laboratorio clínico: son artículos que, utilizando una metodología científica, informan sobre aspectos de laboratorio clínico, tales como: nuevos procedimientos, modificación de técnicas, obtención de valores de referencia para la población colombiana, sensibilidad antimicrobiana, resultado de encuestas serológicas, evaluación de inmunizaciones,etc.

e. Artículos de investigación epidemiológica: son trabajos que, utilizando una metodología científica, dan información sobre problemas de brotes epidémicos, evolución de epidemias, vigilancia y control, y que van más allá de informes susceptibles de publicación en el boletín epidemiológico del instituto.

f. Comunicaciones breves: son trabajos derivados de una investigación básica, epidemiológica o clínica, cuyo conocimiento rápido se considera de gran importancia. 
g. Informes técnicos: son documentos derivados de una reunión de expertos sobre un tema específico.

h. Actualizaciones: son trabajos que, apoyados en bibliografía reciente, destacan los avances más significativos de un problema específico.

i. Memoranda: son documentos muy sobrios que exponen la situación de un aspecto específico.

j. Revisión de temas: son publicaciones densas que revisan exhaustivamente un tema determinado con la más amplia bibliografía posible.

k. Comentarios bibliográficos: son escritos breves, críticos, sobre libros de biomedicina recientemente publicados que, por su impacto, merecen ser comentados.

I. Cartas al editor: son cartas de los lectores solicitando aclaraciones o presentando comentarios sobre material publicado en la revista.

3. Todo material propuesto para publicación será revisado por el Comité Editorial. El editor informará a los autores sobre la recepción de los trabajos y sobre la decisión final que se tome.

4. La revista se reservará el derecho de aceptar o rechazar los artículos y podrá hacer sugerencias que tiendan a mejorar su presentación. Para un mejor cumplimiento de esta función, el Comité Editorial consultará a especialistas en la materia.

5. Los originales de los artículos publicados permanecerán en los archivos de la revista; aquéllos no aceptados, serán devueltos a sus autores.

6. El autor principal recibirá, libre de costo, 5 ejemplares de la revista y 20 separatas de su trabajo.

7. Todo trabajo que se desee publicar en la revista Biomédica deberá ser enviado al editor de la revista, Oficina de Publicaciones, Instituto Nacional de Salud, Avenida Eldorado con carrera 50, zona 6 o Apartado aéreo 80080 y 80334, Santafé de Bogotá, D.C., Colombia.

8. Biomédica sólo considerará para publicación aquellos trabajos que llenen los siguientes requisitos:

a. Ser escritos a máquina, doble espacio, papel tamaño carta, páginas numeradas, dejando un margen de $4 \mathrm{~cm}$ a la izquierda y $2 \mathrm{~cm}$ a la derecha, o en computador llenando las mismas especificaciones y, además, enviando el diskette.

b. Presentar un (1) original y dos (2) copias.

c. El trabajo debe venir organizado así:

- Hoja separada con un título conciso. Puede tener un subtítulo. Autores con nombre, inicial del segundo nombre y apellidos, seguido de un asterisco que indicará al pie de la página, la posición académica, institución, ciudad y país.

- En hoja separada, un resumen en español en donde se enfaticen los aspectos más relevantes del trabajo y que no sea mayor de 250 palabras.

- En hoja separada, un resumen en inglés que tenga el mismo contenido del resumen en español.

- Contenido: introducción, materiales y métodos, resultados, discusión, agradecimientos y bibliografía.

- Las fotografías deben venir en blanco y negro en papel brillante. Biomédica publicará fotografías en colores, cuando a juicio del Comité Editorial, sea necesario. Los cuadros, 
gráficos y figuras deben venir en dibujo en tinta negra o en impresión láser en tamaño proporcional: para una columna, $7,7 \mathrm{~cm}$ de ancho y, para media página, $7,7 \mathrm{~cm} \times 10,3 \mathrm{~cm}$ o página entera, $15,5 \mathrm{~cm} \times 20,5 \mathrm{~cm}$ y, en hoja separada, la leyenda correspondiente.

- La bibliografía debe venir señalada en el texto secuencialmente, con números que correspondan al listado final. Para la correcta transcripción de la bibliografía, Biomédica se acoge a los requisitos unificados para trabajos presentados a revistas biomédicas del Comité Internacional de Editores de Revistas Médicas: Committee of Medical Journal Editors. Uniform requirements for manuscripts submitted to biomedical journals. JAMA 1993; 269:2282-6.

- Deben tenerse en cuenta los siguientes aspectos: en artículos de más de seis autores, se citan sólo los seis primeros autores seguidos de las palabras latina et al., el título completo del artículo, el nombre abreviado de la revista, el año, el volumen, la primera página y el último dígito de la última página.

A manera de ejemplo, citamos:

Goate AM, Haynes AR, Owen MJ, Farrall M, James LA, Lai LY, et al. Predisposing locus for Alzheimer's disease on chromosome 21. Lancet 1989;1:352-5.

Weinstein L, Swartz MN. Pathologic properties of invading microorganism. In: Sodeman WA Jr, Sodeman WA, editors. Pathologic physiology: mechanisms of disease. Philadelphia: Saunders, 1974:457-72.

Renal system (computer program). MSDOS version. Edwardsville (KS): MediSim, 1988. 